\title{
Alternate Approach to the Calculation of Thermal Response Factors for Vertical Borehole Ground Heat Exchanger Arrays Using an Incomplete Bessel Function
}

\author{
Andrew D. Chiasson
}

\author{
Rodwan Elhashmi
}

\begin{abstract}
This article presents yet another methodology for the calculation of dimensionless thermal response factors for vertical borehole ground heat exchanger (GHX) arrays, which is a concept introduced by Eskilson (1987). The presented method is based on a well-known solution to an analogous problem in the field of well bydraulics. This solution method, known mathematically as an incomplete Bessel function, and known in the field of well bydraulics as the 'leaky aquifer function', describes the hydraulic head distribution in an aquifer with predominantly radial flow to a well combined with vertical 'leakage' from geologic layers above and below the pumped aquifer. The solution is adapted to model beat transfer from an array of arbitrarily-placed vertical boreholes of finite depth. With proper expression of parameters in the incomplete Bessel function, we show that g-functions of previous researchers can be approximated. The proposed method has been implemented into Matlab and Excel/ VBA for g-function generation and monthly GHX simulation.
\end{abstract}

\section{INTRODUCTION}

The concept of thermal response factors for application to ground heat exchanger (GHX) dimensioning was originally introduced by Eskilson (1987). Those thermal response factors, known as $g$-functions, were developed using a combination of numerical and analytical techniques with the boundary condition of uniform borehole wall temperature along the length of the boreholes. More recently, based on desire to improve the computational speed of vertical GHX simulation and optimization calculations, several researchers have developed analytical solutions to generate thermal response factors. Such analytical solutions are also more desirable in applications with variablyspaced boreholes, as with underground thermal energy storage systems, for example.

Thermal response factors developed subsequently to those of Eskilson (1987) have been developed under differing boundary conditions at the borehole wall, and the literature now appears unsettled as to the most appropriate boundary condition. In reality, boreholes in a typical vertical GHX design have a common inlet fluid temperature, and both the temperature and heat transfer rate will vary along the length of the boreholes. Cimmino and Bernier (2014) succinctly summarize the various types of boundary conditions possible in development of thermal response factors: (i) uniform heat extraction rate along the length of the boreholes such that heat extraction rates are equal for all boreholes and the average temperatures along the length of all boreholes are unequal, (ii) uniform heat extraction rate along the length of the boreholes such that the average temperature along the length of the boreholes is equal for all 
boreholes, and (iii) uniform borehole wall temperature along the length of the boreholes such that the borehole wall temperature is equal for all boreholes (Eskilson's method).

The purpose of the present research is to approach the development of dimensionless temperature response factors from a different perspective by adapting a solution to an analogous problem in the field of well hydraulics. Results are compared to those of other published solutions.

\section{BACKGROUND AND LITERATURE REVIEW}

Eskilson (1987) introduced the concept of $g$-functions for heat extraction (or rejection) boreholes. The $g$ functions provide a relation between the heat extracted from the ground at the borehole wall $q^{\prime}$ and the borehole wall temperature $T_{b}$ as:

$$
T_{b}=T_{g}-\frac{q^{\prime}}{2 \pi k} g\left(\frac{t}{t_{s}}, \frac{r_{b}}{H}, \frac{B}{H}\right)
$$

where $t_{s}$ is a characteristic time scale given by $H^{2} /(9 \alpha)$. As described by Cimmino et al. (2013), Eskilson's approach introduced a fourth non-dimensional parameter, $D / H$, but that parameter was not explicitly varied in his studies, as he used a fixed value of $D$ equal to 4 or $5 \mathrm{~m}$.

Eskilson's $g$-functions have been thoroughly described in the literature (eg. Cimmino et al. (2013), Cimmino and Bernier (2014), and Spitler and Bernier (2016)), and are often taken as a benchmark for comparison with other thermal response factors. Cimmino et al. (2013) describe the numerous analytical solution methods attempting to duplicate Eskilson's work, but an exact duplication has been elusive due to the method of the spatial superpositioning of single borehole solutions used by Eskilson. He generated thermal response factors numerically using twodimensional (radial and axial) numerical simulations combined with spatial superposition to effectively obtain a threedimensional response of the borehole heat exchanger field; the temperature at the borehole walls was uniform along the length of the boreholes and equal for all boreholes.

Cimmino et al. (2013) proposed a new method to approximate $g$-functions. The method, based on the analytical finite line source, accounts for the variation of heat extraction rates among boreholes due to thermal interaction among boreholes and for the $\mathrm{D} / \mathrm{H}$ ratio. The heat extraction rates obtained with this method showed good agreement with Eskilson's numerical model, but there are some differences that were explained by the fact that the methods used different boundary conditions at the borehole wall. However, for small simulation times, the differences are small and the response factors are almost identical for up to 10 and 6 years for $3 \times 2$ and $10 \times 10$ borehole fields, respectively. For large borehole fields, however, thermal interactions among boreholes become important and the observed differences between the Cimmino et al. (2013) model and Eskilson's $g$-function increase. For example, the $g$-function of a $10 \times 10$ borehole field obtained with the Cimmino et al. (2013) method overestimates the $g$-function by $32 \%$ at steady-state.

Cimmino and Bernier (2014) were able to closely match Eskilson's $g$-functions by dividing boreholes into segments to consider the variation of the heat extraction rates along the length of the boreholes, and then applying the analytical finite line source (FLS) solution to calculate the temperature variations at the wall of each borehole segment along the axial direction. Their method accounted for the time variation of the heat extraction rates among boreholes and along the length of individual boreholes to obtain a uniform borehole wall temperature equal for all boreholes in accordance with the original boundary conditions proposed by Eskilson. The difference between the Cimmino and Bernier (2014) model was within 5\% of Eskilson's $g$-functions for all studied bore fields, except for fields of boreholes located on a single row. 


\section{PROPOSED MODEL}

\section{Conceptual Model}

A conceptual diagram of a boreole heat exchanger is shown in Figure 1. The term borehole heat exchanger (BHE) refers to a closed-loop pipe assembly installed in a vertical borehole with radius $r_{b}$ over some active depth $H$ for purposes of heat exchange with the subsurface. The top of the active BHE is buried at some depth $(D)$ from the ground surface, and typical constructions consist of a single U-tube grouted in a borehole as shown in Figure 1, but other geometries exist (i.e., double U-tube, concentric tube, and groundwater-filled boreholes). These boreholes are designed to extract (or reject) a certain amount of thermal energy $(q)$ per unit depth $(H)$ by pumping a fluid through the heat exchanger. Thus, $q^{\prime}$ is the heat extraction or rejection rate divided by the active borehole depth. Heat transfer occurs from the fluid to the ground whose undisturbed "far-field" temperature remains at $T_{g}$. $T_{b}$ represents the average temperature at the borehole wall.

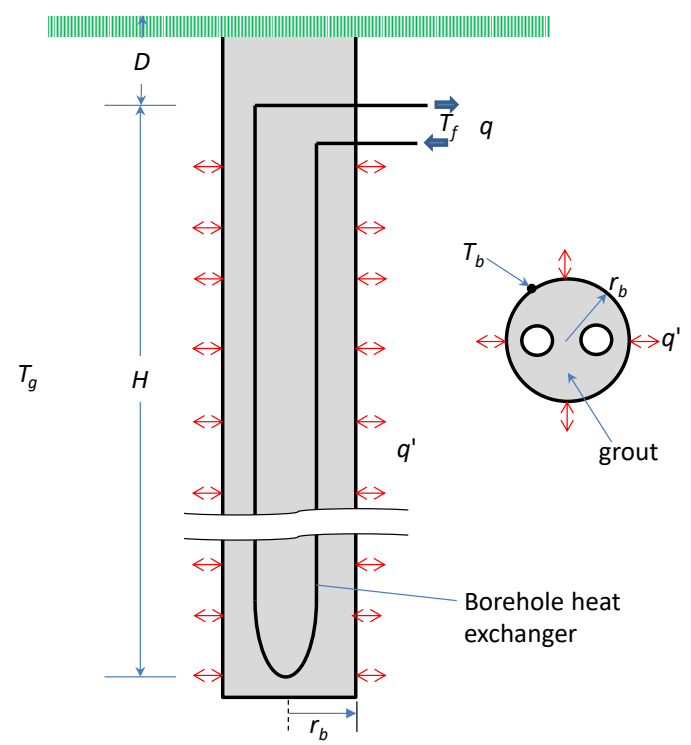

Figure 1. Conceptual diagram of a borehole heat exchanger.

Coupled to a building or other time-varying thermal load, multiple (i.e., two to tens) BHEs are connected together in a collective array to form what we refer here to as a ground heat exchanger (GHX). A common GHX design configuration is a line or grid pattern of BHEs with a fixed borehole-to-borehole field spacing, B. Over time, BHEs become thermally-interacting.

\section{Single Borehole}

Governing Equations: The simple case of one-dimensional, radial heat conduction is given by the following partial differential equation:

$$
\frac{\partial^{2} T}{\partial r^{2}}+\frac{1}{r} \frac{\partial T}{\partial r}=\frac{1}{\alpha} \frac{d T}{d t}
$$

In an analogy to heat conduction, Equation 2 is used in the field of well hydraulics to describe radial flow toward a well. However, in many situations, flow toward a well consists of vertical flow components caused by 'leakage' from 
overlying and/or underlying geologic layers. The partial differential equation describing such cases is given by:

$$
\frac{\partial^{2} T}{\partial r^{2}}+\frac{1}{r} \frac{\partial T}{\partial r}+\xi=\frac{1}{\alpha} \frac{d T}{d t}
$$

where $\xi$ expresses the ratio of vertical to horizontal thermal (or hydraulic) transmissivities or $\Delta T\left(\alpha t_{s}\right)^{-1}$.

Initial and Boundary Conditions: The initial condition is prescribed as $T(r, t)=T(r, 0)=T_{g}$. The boundary conditions are given as $T(r, t)=T(\infty)=T_{g}$, and $\lim _{r \rightarrow 0}\left(r \frac{\partial T}{\partial r}\right)=\frac{q^{\prime}}{2 \pi k}$.

Solution and Implementation: The solution to Equation (2) for the temperature change $(\Delta T)$ in a heatconducting medium is that around an infinite line source, and is well known as:

$$
\Delta T=\frac{q^{\prime}}{4 \pi k} \int_{u}^{\infty} \frac{e^{-u}}{u} d u=\frac{q^{\prime}}{4 \pi k} \cdot \operatorname{Ei}(u)=\frac{q^{\prime}}{4 \pi k} \cdot W(u)
$$

where $E i$ is known mathematically as the exponential integral function, $W$ is known in the field of well bydraulics as the Theis well function or more simply as the well function, and $u=r^{2} /(\alpha t)$.

The solution to Equation 3, which models the temperature distribution around a finite line source, is:

$$
\Delta T=\frac{q^{\prime}}{4 \pi k} \int_{u}^{\infty} \frac{1}{u} \exp \left(-u-\frac{r^{2}}{4 \beta^{2} u}\right) d u=\frac{q^{\prime}}{4 \pi k} \cdot W\left(u, \frac{r}{\beta}\right)
$$

where $W(u, r / \beta)$ is known mathematically as an Incomplete Bessel Function, and in the field of well bydraulics as the leaky well function or the Hantush-Jacob well function after Hantush and Jacob (1955), and $\beta$ is a 'leakage factor'. Further mathematical details of these functions are described by Harris (2008).

In an analogy to heat conduction, we define $\beta$ as $\left(\alpha t_{s}\right)^{1 / 2}$, where $t_{s}$ is the characteristic time scale defined by Eskilson (1987) as $H^{2} /(9 \alpha)$. Thus, Equation 5 can be adapted to model the temperature distribution around a finite line source with length $H$ and burial depth $D$ as:

$$
\Delta T=\frac{q^{\prime}}{4 \pi k} \cdot W(u, b)
$$

where we define:

$$
b=3 \frac{r}{H}\left(1+\frac{D}{H}\right)^{-1}
$$

The formulation for $g$ is now expressed with the leaky well function notation as:

$$
g\left(\frac{t}{t_{s}}, \frac{r_{b}}{H}, \frac{B}{H}, \frac{D}{H}\right)=\frac{1}{2} W(u, b)
$$




\section{Multi-Borehole Arrays}

The inherent three-dimensional temperature distribution in the ground is modeled by superpositioning of the single-borehole solution in two different ways corresponding to boundary condition (i) and boundary condition (iii) described above. That is: (i) uniform heat extraction rate along the length of the boreholes such that heat extraction rates are equal for all boreholes and the average temperatures along the length of all boreholes are unequal, and (iii) uniform borehole wall temperature along the length of the boreholes such that the borehole wall temperature is equal for all boreholes (Eskilson's method).

By spatial superpositioning of finite line sources calculated with the proposed method, the response factors are determined for any arbitrary borehole field pattern and not necessarily limited to the prescribed geometries of Eskilson. Spatial superpositioning involves calculation of the dimensionless temperature response $(g)$ in each borehole due to all the others at a particular time. Thus, the dimensionless temperature response of a particular borehole $\left(g_{b}\right)$ is the sum of the influence from all other boreholes:

$$
g_{b x, y}=\sum_{i=1}^{x b o r e s} \sum_{j=1}^{y b o r e s} g_{i, j}
$$

where $i$ and $j$ are indices, and $x$ bores and $y$ bores are the number of boreholes in the $x$ and $y$ direction, respectively. The $g$-function can be calculated for an entire borehole field at a particular time dividing the result of Equation 9 by the number of boreholes in the field.

Development of $g$-functions with the proposed method using boundary condition (i) is straightforward: an arbitrary constant heat rate was specified per borehole, and the temperature change at the borehole walls were calculated directly and then non-dimensionalized. Development of $g$-functions with the proposed method using boundary condition (iii) is less straightforward. Here, we specified a temperature change at each borehole and applied a multivariable optimization method to iteratively adjust the heat rates per length per borehole such that the borehole wall temperature remained unchanged. Calculation times using boundary condition (iii) were orders of magnitude greater than calculation times using boundary condition (i).

\section{RESULTS AND DISCUSSION}

To the best of our knowledge, Eskilson (1987) used a total borehole depth $(D+H)$ of $115 \mathrm{~m}$ in development of his $g$-functions. The active borehole length $(H)$ was $110 \mathrm{~m}$ and the buried depth $(D)$ was $5 \mathrm{~m}$. In comparison to Eskilson's work in what follows, we used a $D / H$ ratio equal to 0.0454 and an $r_{b} / H$ ratio of 0.0005 .

\section{Comparison to Eskilson's g-functions - The Case of Constant Uniform Heat Rate Along the Borehole Lengths}

$g$-functions determined with the proposed method using the boundary condition of uniform heat extraction rate along the length of the boreholes are compared to those of Eskilson (1987) in Figure 2. $g$-functions were generated for a variety of borehole field configurations (L-shaped, U-shaped, linear, open-square, rectangular, and square).

Observations of the comparisions of the $\mathrm{g}$-functions shown in Figure 2 are consistent with those of other researchers (eg. Cimmino et al. (2013) and Malayappan and Spitler (2012)). That is, analytical solutions using a constant heat flux boundary condition at the borehole wall are essentially identical to Eskilson's $g$-function for a single borehole, but tend to over-predict Eskilson's g-functions for borehole fields with increasingly lower $B / H$ ratio and increasingly greater number of boreholes. Here, we also observe that the proposed solution with increasing $B / H$ ratio eventually begins to under-predict Eskilson's $g$-functions. We further observe some differences in the $g$-functions obtained with differing boundary conditions as a function of $\ln \left(t / t_{s}\right)$. These deviations imply different handling of end effects of the boreholes, which previous researchers attributed to the differing boundary conditions at the borehole wall. 

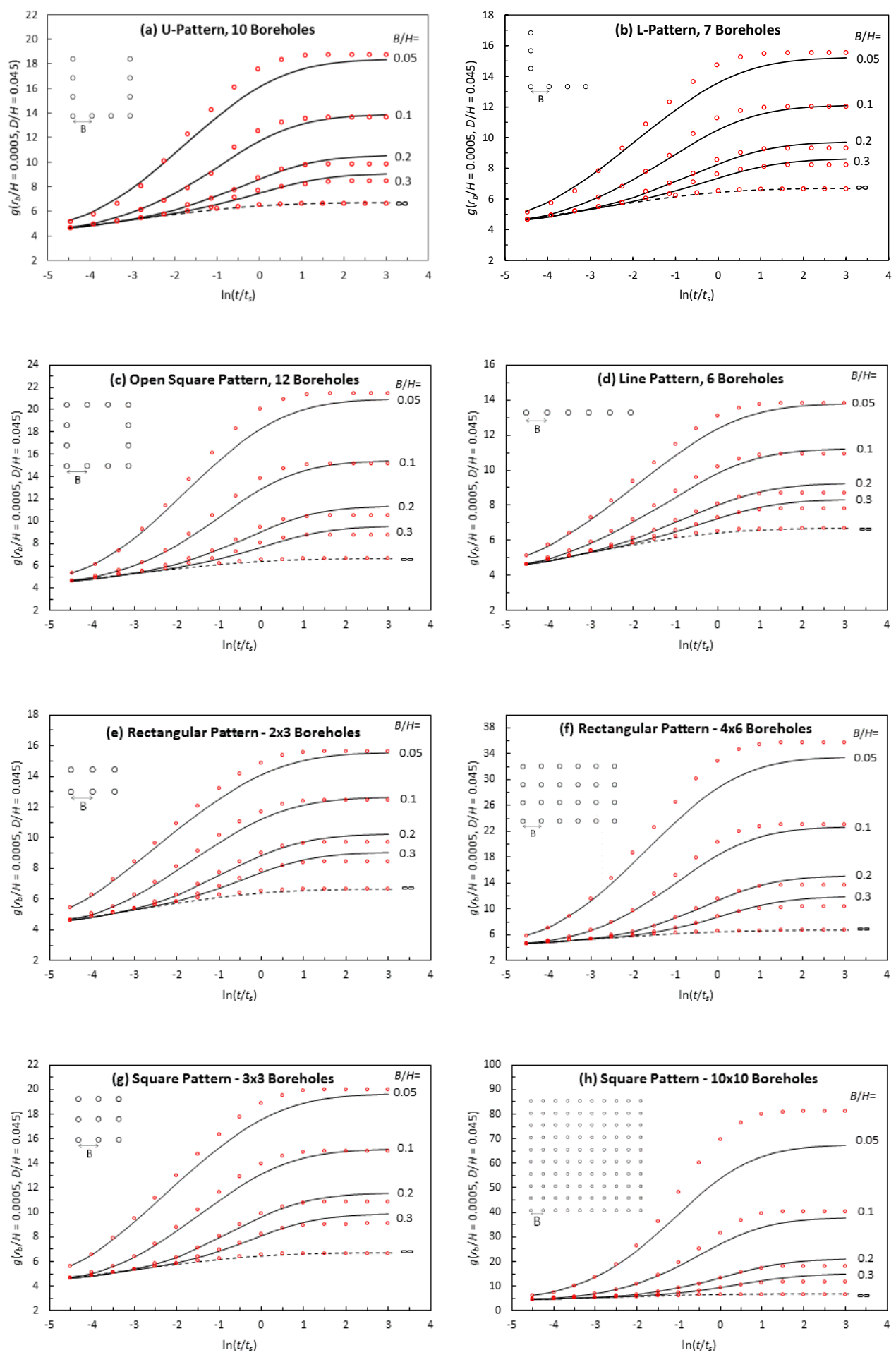

Figure 2. Comparison of g-functions calculated by proposed model to those of Eskilson (1987) for various borehole field configurations using a boundary condition of uniform heat extraction rate along the length of the boreholes. Note $B / H=\infty$ is the $g$-function for a single borehole. 


\section{Comparison to Eskilson's g-functions - The Case of Constant Borehole Wall Temperature}

$g$-functions determined with the proposed method using the boundary condition of constant borehole wall temperature are compared to those of Eskilson (1987) in Figure 3. These $g$-functions were generated for comparison only for densely-packed borehole field configurations (i.e., $B / H$ ratio of 0.05 ). Here, we observe a much closer match between the proposed method and Eskilson's g-functions for densely-packed borehole fields with low $B / H$ ratio. The steady-state values of the $g$-function determined by the proposed method are within $4 \%$ of Eskilson's values.

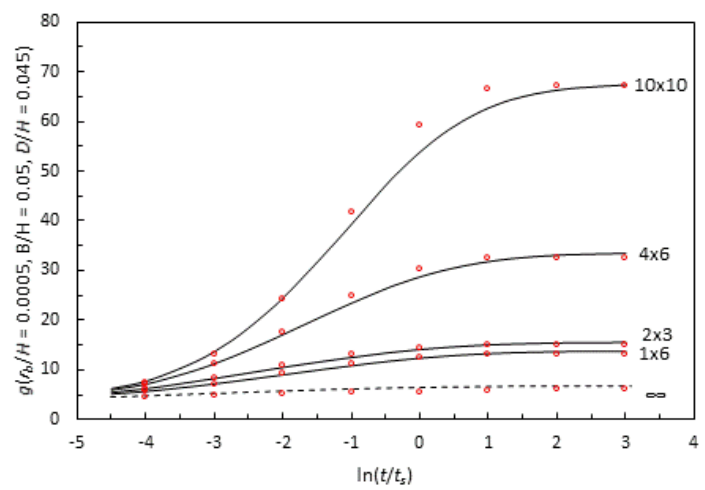

Figure 3. Comparison of $g$-functions calculated by proposed model to those of Eskilson (1987) for various borehole field configurations using a boundary condition of constant temperature along the length of the boreholes. Note $B / H=\infty$ is the $g$-function for a single borehole.

\section{Comparison to Other Solution Methods}

$g$-functions generated with the proposed method using the boundary condition of constant heat flux at the borehole wall are compared to other solution methods graphically in Figure 4. These solution methods, described by Cimmino et al. (2013), are: (a) the analytical finite line source method and (b) the method developed by Cimmino et al. (2013). The former uses boundary condition (i) described above, while the latter uses boundary condition (ii) described above.
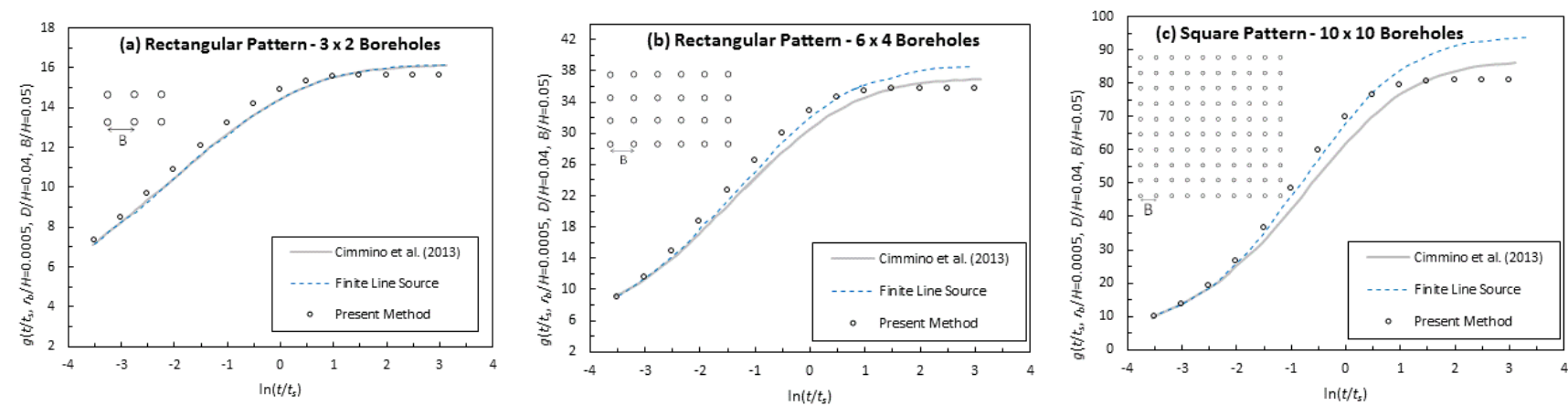

Figure 4. Comparison of $g$-functions calculated by proposed model to those presented by Cimmino et al. (2013) for boreholes in a (a) 3x2 rectangular pattern, (b) 6x4 rectangular pattern, and (c) 10x10 square pattern.

A review of Figure 4 reveals that the proposed solution matches the finite line source model up to a time of $\ln \left(t / t_{s}\right)=0$, after which time the proposed solution more closely agrees with the method developed by Cimmino et al. (2013). 


\section{Discussion of Practical GHX Design Sizing}

Here, we provide a comparison of borehole depth calculations for a reference building, where the GHX is designed using (i) the proposed method for the case of constant uniform heat rate along the borehole lengths, and (ii) the $g$-functions of Eskilson (1987). Loads for a reference medium-sized office building in Boulder, CO (U.S. Department of Energy Climate Zone 5B) were taken from datasets described by Field et al. (2010). The peak heating and cooling loads are $239 \mathrm{~kW}$ and $350 \mathrm{~kW}$, respectively, and the annual cooling-to-heating loads ratio is about 1.6.

While GHX designs have many specific considerations, some generalizations are noted and made use of here. In most U.S. GHX designs, $H \approx 75 \mathrm{~m}$ and $B \approx 6 \mathrm{~m}$, which results in $B / H \approx 0.08$. Further, given the general relationship between thermal conductivity to density and heat capacity, for common geologic materials, $\alpha \approx 1 \times 10^{-6}$ $\mathrm{m}^{2} / \mathrm{s}$. The foregoing generalities result in $\ln \left(t / t_{s}\right) \approx 0$ for a 20 -year GHX design time scale.

In sizing a 10x10 borehole GHX with $B / H=0.1$ for the reference medium-sized office building in Boulder, $\mathrm{CO}$ using the foregoing design assumptions, we find a difference of $\sim 2 \%$ between the design length calculated with $g$ functions derived from the proposed method versus Eskilson's $g$-functions. For an unrealistically close borehole spacing of $B / H=0.05$, the difference between the two methods is $\sim 8 \%$, which is significantly less than might be implied by observation of Figure 2(h). The main reasons for the similarity in the GHX design length determined by both methods is due to the inherent design considerations and building loads profile. Choice of $B$ and $H$ affect time scales of the problem, while the building loads dictate the short- and long-term ground thermal response. Thus, in a building with perfectly balanced annual loads, the long-term ground thermal response would be negligible, and both methods would yield nearly identical results.

\section{CONCLUSION}

This article has introduced yet another methodology for the calculation of dimensionless thermal response factors for vertical GHXs. The method, based on a well-known solution to an analogous problem in the field of well hydraulics, is known mathematically as an incomplete Bessel function, and known in the field of well hydraulics as the 'leaky aquifer function'. The method has been implemented into Matlab and Excel/VBA for computationally-efficient $g$-function generation and subsequent GHX design sizing and/or simulation of thermally-interacting BHEs.

$g$-functions generated by the proposed method were compared to those of Eskilson (1987) for several borehole field configurations (L-shaped, U-shaped, linear, open-square, rectangular, and square) under two boundary conditions at the borehole wall (constant heat flux and constant temperature). As concluded by previous researchers, $g$-functions developed under the varying borehole wall boundary conditions have some similarities and some important differences. In reality, boreholes in a vertical borehole field have a common inlet fluid temperature, and both the temperature and heat transfer rate will vary along the length of the boreholes. Future work consisting of experimental validation of the various boundary condition assumptions would be quite helpful.

\section{NOMENCLATURE}
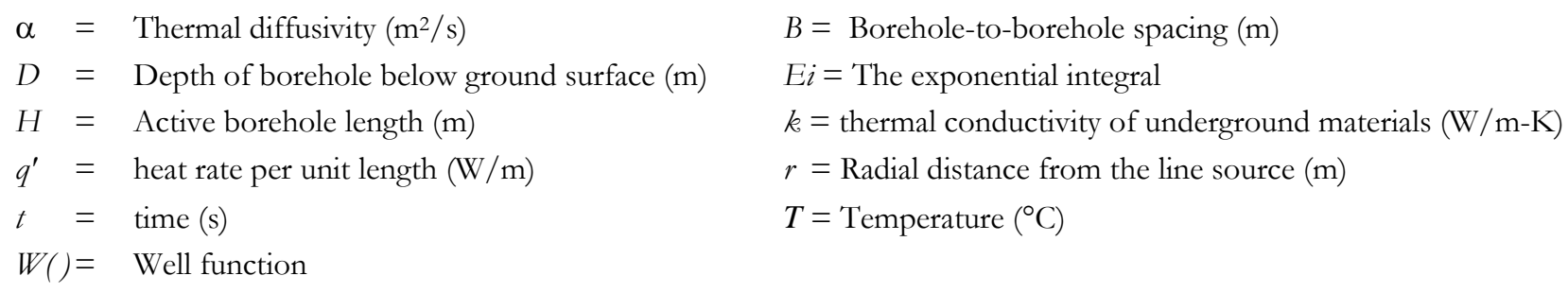

\section{Subscripts}

$b=$ borehole,$\quad g=$ ground,$\quad s=$ surface, characteristic time-scale 


\section{REFERENCES}

Cimmino, M., and M. Bernier. 2014. A semi-analytical method to generate g-functions for geothermal bore fields. International Journal of Heat and Mass Transfer, 70(2014): 641-650.

Cimmino, M., M. Bernier, and F. Adams. 2013. A contribution towards the determination of g-functions using the finite line source. Applied Thermal Engineering, 51(2013): 401-412.

Eskilson, P., 1987. Thermal Analysis of Heat Extraction Boreboles. Thesis (PhD), University of Lund, Lund (1987) 264 pp.

Field, K., Deru, M., and Studer, D., 2010. Using DOE Commercial Reference Buildings for Simulation Studies. Presented at SimBuild 2010 New York, New York, August 11-13, 2010. http://en.openei.org/datasets/files/961/pub/

Hantush, M.S., and C.E. Jacob, 1955. Nonsteady radial flow in an infinite leaky aquifer. Transactions of the American Geophysical Union, $36: 95-100$.

Harris, F.E. 2008. Incomplete Bessel, generalized incomplete gamma, or leaky aquifer functions. Journal of Applied and Computational Ma thematics 215(1): 260-269.

Malayappan, V., J.D. Spitler. 2013. Limitation of using uniform heat flux assumptions in sizing vertical borehole beat exchanger fields. Proceedings of Clima 2013. June 16-19. Prague

Spitler, J.D. and M. Bernier. 2016. Vertical borehole ground heat exchanger design methods. In S.J. Rees Advances in Ground-Source Heat Pump Systems. London: Woodhead Publishing. 